\title{
Modificação no Posicionamento do Paciente para o Procedimento para Prolapso e Hemorroidas (PPH): Decúbito Ventral com Coxim e Membros Inferiores Afastados
}

\author{
The Use of a Modified Jacknife Position for Stapled \\ Hemorroidopexy Procedure (PPH)
}

\author{
FABIO VIEIRATEIXEIRA ${ }^{1}$; ROGÉRIO SAAD-HOSSNE²; \\ PAULOTEIXEIRAJÚNIOR ${ }^{3}$ TSBCP)
}

\begin{abstract}
${ }^{1}$ Especialista em Coloproctologia pela Sociedade Brasileira de Coloproctologia, Professor Voluntário do Departamento de Cirurgia e Ortopedia e do Ambulatório de Doenças Inflamatórias Intestinais da Faculdade de Medicina - UNESP Botucatu e Médico da UNIGASTRO, Marília; ${ }^{2}$ Professor Assistente Doutor do Departamento de Cirurgia e Ortopedia - UNESP Botucatu; ${ }^{3}$ Médico(a) da UNIGASTRO, Marília - São Paulo - Brasil.
\end{abstract}

TEIXEIRA FV; SAAD-HOSSNE R; TEIXEIRA JUNIOR P. Modificação no Posicionamento do Paciente para o Procedimento para
Prolapso e Hemorroidas (PPH): Decúbito Ventral com Coxim e Membros Inferiores Afastados. Rev bras Coloproct, 2008;28(3): $315-318$.

RESUMO: Objetivo: A doença hemorroidária(DH) é prevalente em cerca de $5 \%$ da população brasileira. Os casos mais avançados da DH são tratados com ressecção dos mamilos prolapsados (hemorroidectomia) e fechamento(técnica de Ferguson) ou não da ferida operatória(Miligan Morgan). No entanto, a dor no pós-operatório e o longo período de recuperação dos pacientes submetidos a hemorroidectomia convencional são os principais inconvenientes das técnicas. 0 método da hemorroidopexia ou procedimento para prolapso e hemorróidas(PPH) vem sendo realizado desde 1998, e tem como principal vantagem a resolução da DH com menos dor e recuperação mais rápida do paciente. Nosso objetivo é apresentar uma modificação técnica no posicionamento do paciente com DH que será submetido ao PPH. Métodos e pacientes: Desde Janeiro de 2008 foram operados 5 pacientes no Hospital UNIMAR, Marília, São Paulo. Todos eram portadores de doença hemorroidária avançada - Grau III e IV. Os procedimentos foram realizados com bloqueio raqui-medular em sela com sufentanil associado à bupivacaína. Os pacientes foram posicionados em decúbito ventral com coxim de cerca de $20 \mathrm{~cm}$ de altura colocada na altura da espinha ilíaca ântero-superior. Foram usadas fitas adesivas para afastar lateralmente a região glútea. O cirurgião ficou posicionado no centro, no vão entre os membros inferiores do paciente. $O$ primeiro auxiliar posicionando à direita e a instrumentadora à esquerda. $\mathrm{O}$ canal anal foi dilatado manualmente e fixado o dilatador do PPH. Em todos os pacientes a linha pectínea foi facilmente identificada, e obteve-se a exposição de 3 a $4 \mathrm{~cm}$ do reto acima da linha pectínea. A bolsa foi realizada com fio de polipropileno (Prolene $® 0$ com agulha de $1,5 \mathrm{~cm}$ ) sem a necessidade de utilização do afastador de 2 canas. Os pontos compreenderam a mucosa retal tomando-se cuidado em não incluir a camada muscular do reto. Após o disparo e retirada do aparelho, identificou-se com facilidade a linha de grampos sem a necessidade de colocar qualquer tipo de afastador auxiliar. Em nenhum dos casos foi necessário hemostasia adicional, com pontos aplicados sobre a linha de grampos. Conclusões: Pudemos observar que com esse posicionamento a exposição do canal anal fica mais fácil, sem a necessidade de utilizar qualquer tipo de afastador (tipo 2 canas) quando da confecção da sutura em bolsa. Além disso, com o decúbito ventral, o cirurgião opera em pé e a equipe cirúrgica tem maior mobilidade, o que não acontece quando o paciente está posicionado em litotomia. Ademais, o posicionamento do cirurgião de frente para a região anal facilita a confecção da bolsa, a atadura do nó, o disparo do aparelho e a revisão da linha de grampos.

Descritores: PPH, doença hemorroidária, tratamento cirúrgico.

Trabalho realizado no Serviço de Endoscopia e Cirurgia do Aparelho Digestivo e Coloproctologia - UNIGASTRO / Hospital UNIMAR, Marilia, São Paulo - SP - Brasil. 
Rev bras Coloproct Julho/Setembro, 2008
Modificação no Posicionamento do Paciente para o Procedimento para Prolapso e Hemorroidas (PPH): Decúbito Ventral com Coxim e Membros Inferiores Afastados Fábio Vieira Teixeira e Cols.
Vol. 28

\section{INTRODUÇÃO}

A hemorroidopexia com uso de grampeador intestinal circular ou conhecida no nosso meio por procedimento para prolapso e hemorróidas (PPH) é uma técnica indicada no tratamento da doença hemorroidária (DH) avançada (III e IV graus). Vários estudos randomizados foram realizados nos últimos anos comparando a técnica do PPH com as hemorroidectomias convencionais tipo Ferguson ou Miligan Morgan. A conclusão na maioria desses estudos foi que a técnica do PPH é efetiva quanto a hemorroidectomia convencional no tratamento da $\mathrm{DH}$, com o benefício de levar a menor dor pós-operatória e retorno mais rápido do paciente as suas atividades habituais. ${ }^{1-3}$ A maioria dos autores que realizam a hemorroidopexia, utilizam a posição de litotomia como a de escolha no posicionamento do paciente na mesa operatória..$^{1-5}$ No entanto, em nossa opinião, a posição de litotomia impede que os auxiliares fiquem próximos ao campo operatório o que, em algumas vezes, dificulta o procedimento. Nos Estados Unidos, a posição de decúbito ventral tipo canivete (prone jacknife) é a preferida. Entretanto, o cirurgião se posiciona ao lado do paciente. ${ }^{4} \mathrm{Em}$ nosso serviço todas as operações orificiais são realizadas com o paciente em decúbito ventral com colocação de coxim sob a região de cristas ilíacas ântero-superiores (jacknife). Entretanto, durante nossa experiência inicial com o PPH em 2002, concluímos que a posição de jacknife dificultava muito o procedimento (confecção da bolsa e acionamento do PPH) uma vez que o cirurgião operava ao lado do paciente.

Nosso objetivo com essa nota prévia é o de apresentar uma modificação simples no posicionamento do paciente na mesa operatória que facilita muito o procedimento do $\mathrm{PPH}$.

\section{MÉTODO E PACIENTES}

Desde Janeiro de 2008 foram operados 5 pacientes no Hospital UNIMAR, Marília, São Paulo. Todos eram portadores de doença hemorroidária avançada - Grau III e IV. Os procedimentos foram realizados com bloqueio raqui-medular em sela com sufentanil associado a bupivacaína. Todos pacientes receberam antibioticoprofilaxia com getamicina $(3 \mathrm{mg} / \mathrm{Kg}$ de peso, dose única, por via $\mathrm{EV}$ ) associada a metronidazol (15 a $18 \mathrm{mg} / \mathrm{Kg}$ de peso, $8 \mathrm{em} 8$ horas, por via EV). Os pacientes foram posicionados em decúbito ventral, com coxim de cerca de $20 \mathrm{~cm}$ de altura colocada na altura da espinha ilíaca ântero-superior. Foram usadas fitas adesivas para afastar lateralmente a região glútea. (Figura 1) O cirurgião ficou posicionado no centro, no vão entre os membros inferiores do paciente. $\mathrm{O}$ primeiro auxiliar posicionando à direita e a instrumentadora à esquerda. O canal anal foi dilatado manualmente, e fixado o dilatador do PPH. Em todos os pacientes a linha pectínea foi facilmente identificada, e obteve-se a exposição de 3 a $4 \mathrm{~cm}$ do reto acima da linha pectínea. (Figura 2) A bolsa foi realizada com fio de polipropileno (Prolene ${ }^{\circledR} 0 \mathrm{com}$ agulha de $1,5 \mathrm{~cm}$ ) sem a necessidade de utilização do afastador de 2 canas. (Figura 3) Os pontos compreenderam a mucosa retal tomando-se cuidado em não incluir a camada muscular do reto. Após o disparo e retirada do aparelho identificou-se com fa-

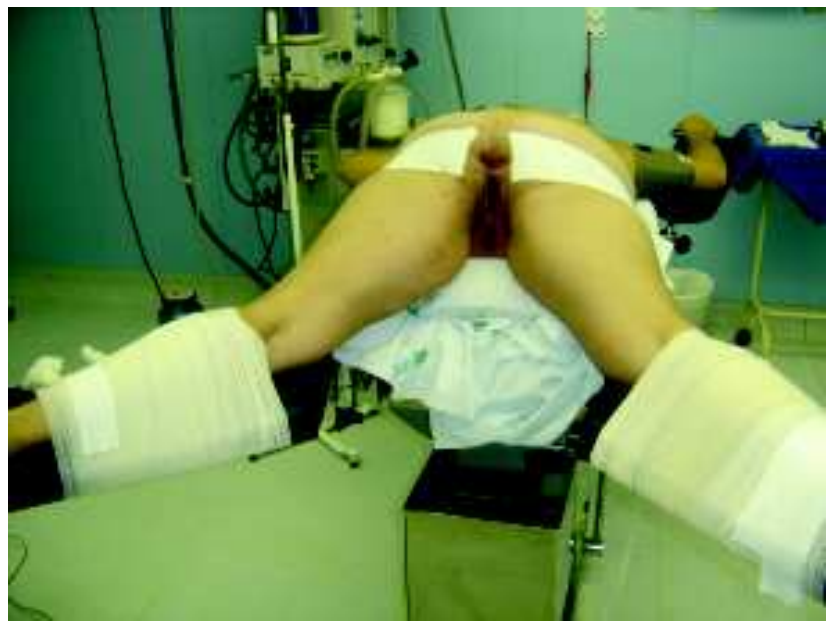

Figura 1 - Paciente em decúbito ventral com coxim e membros inferiores afastados.

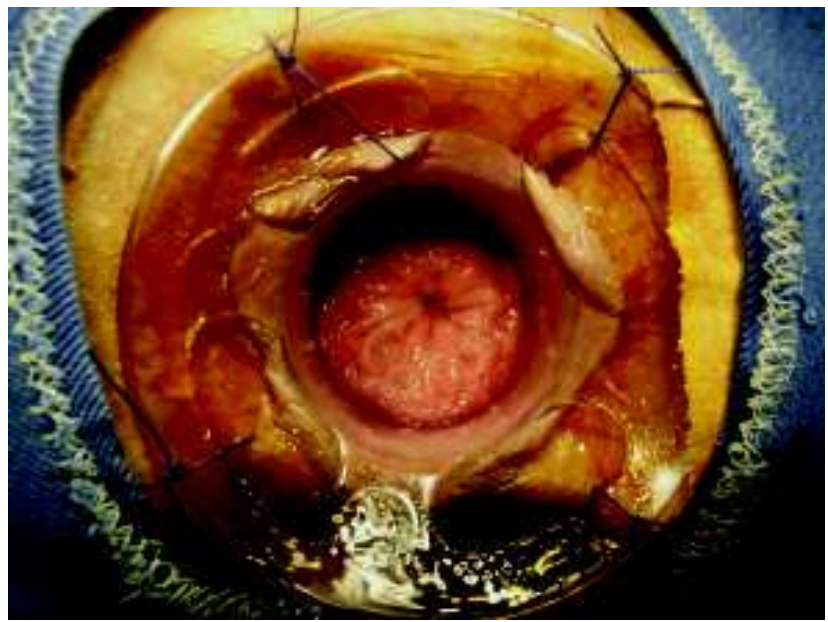

Figura 2 - Mucosa do reto baixo $4 \mathrm{~cm}$ acima da linha pectínea. 
Rev bras Coloproct Julho/Setembro, 2008
Modificação no Posicionamento do Paciente para o Procedimento para Prolapso e Hemorroidas (PPH): Decúbito Ventral com Coxim e Membros Inferiores Afastados Fábio Vieira Teixeira e Cols. cilidade a linha de grampos sem a necessidade de colocar qualquer tipo de afastador auxiliar. (Figura 4) Em nenhum dos casos foi necessário hemostasia adicional com pontos aplicados sobre a linha de grampos. Os pacientes receberam alta 15 a 18 horas após o procedimento, e nenhum tipo de complicação pós-operatória foi observada nessa série de casos.

\section{CONCLUSÃO}

Em nossa opinião, em pacientes portadores de doença hemorroidária avançada, quando da execução do procedimento para prolapso e hemorróidas, a posição de jacknife com membros inferiores afastados

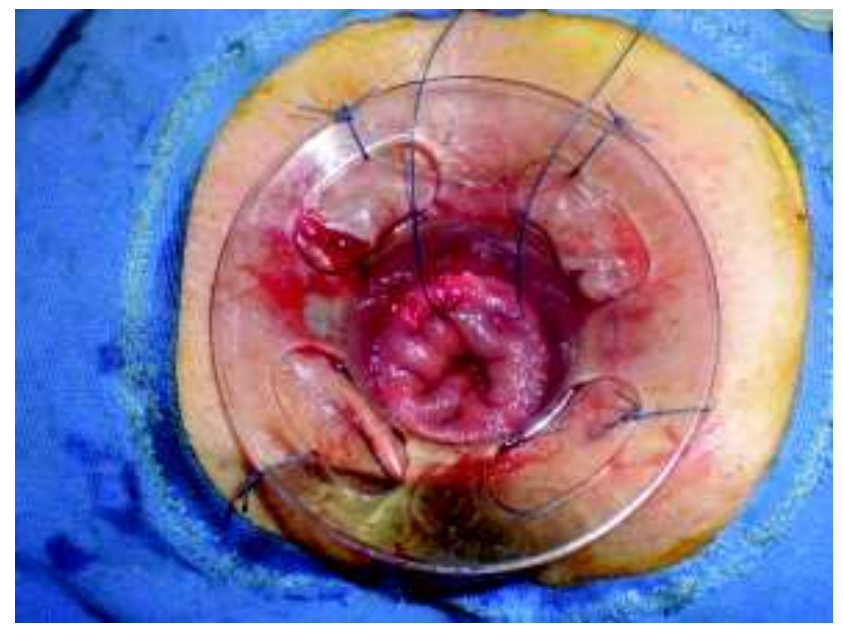

Figura 3 - Pontos da sutura em bolsa 4 cm acima da linha pectínea. permite ao cirurgião operar em posição ortostática e de frente para a região perineal. Todo o ato operatório é facilitado, uma vez que os auxiliares ficam confortavelmente posicionados ao lado do paciente e podem ajudar adequadamente no procedimento, o que não ocorre com o paciente em litotomia. Além disso, a posição de jacknife permite uma ampla exposição do canal anal e de alguns centímetros do reto baixo não sendo necessário nenhum tipo de afastador, além do espéculo anal que vem no kit do PPH. Tal exposição facilita a confecção de uma bolsa sob visão direta e, após o disparo do aparelho, a identificação da sutura de grampos sem a necessidade de qualquer tipo de afastador.

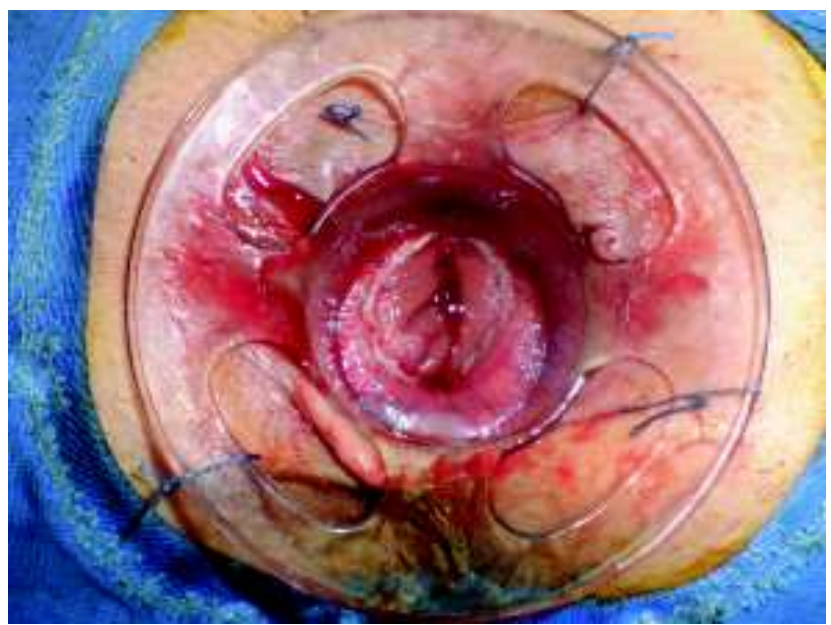

Figura 4 - Linha de grampos posicionada $3 \mathrm{~cm}$ acima da linha pectínea.

\begin{abstract}
Our aim is to present a modification in patient placement on surgical table for the treatment of prolapsed hemorrhoids by using PPH technique. We propose a modified jackknife position for PPH procedure where the surgeon stays between patient's legs. The modified jackknife position may provide better surgical field with better view of the dentate line which facilitates pursestring confection. In Brazil, the majority of the surgeons prefer lithotomic position for PPH technique based on Longo's first reports. In our opinion, modified jackknife position provides both better surgical field for pursestring placement and better view of the staple line after PPH firing.
\end{abstract}

Key words: PPH; Hemorrhoids; Surgical Treatment.

\section{REFERÊNCIAS}

1. Wong JC, Chung CC, Yau KK, Cheung HY, Wong DC, Chan OC, Li MK. Stapled technique for acute thrombosed hemorrhoids: a randomized, controlled trial with long-term results. Dis Colon Rectum. 2008;51(4):397-403
2. Kraemer M, Parulava T, Roblick M, Duschka L, Müller-Lobeck H. Prospective, randomized study: proximate PPH stapler vs. LigaSure for hemorrhoidal surgery. Dis Colon Rectum. 2005;48(8):1517-22.

3. Shao WJ, Li GC, Zhang ZH, Yang BL, Sun GD, Chen YQ. Systematic review and meta-analysis of randomized controlled 
Rev bras Coloproct

Julho/Setembro, 2008
Modificação no Posicionamento do Paciente para o Procedimento para Prolapso e Hemorroidas (PPH): Decúbito Ventral com

Vol. 28 $\mathbf{N}^{\mathbf{0}} 3$

Coxim e Membros Inferiores Afastados

Fábio Vieira Teixeira e Cols.

trials comparing stapled haemorrhoidopexy with conventional haemorrhoidectomy. Br J Surg. 2008;95(2):147-60

4. Sobrado CW, Cotti GC, Coelho FF, Rocha JR. Initial experience with stapled hemorrhoidopexy for treatment of hemorrhoids. Arq Gastroenterol. 2006;43(3):238-42

5. Nahas SC, Borba MR, Brochado MC, Marques CF, Nahas CS, Miotto-Neto B. Stapled hemorrhoidectomy for the treatment of hemorrhoids. Arq Gastroenterol. 2003;40(1):35-9.

\section{Endereço para correspondência:}

PROF. DR. FABIO V. TEIXEIRA - UNIGASTRO

Rua Dr. Próspero Cecílio Coimbra, 80 - Cidade Universitária Marilia - São Paulo - Brasil

CEP 17525-160

TEL / FAX +55 14 2105-4562

E-mail fabioteixeira@unimedmarilia.com.br 